\title{
Lenticular galaxies in the process of evolution
}

\author{
S. N. Kemp ${ }^{1,2}$, J. A. Pérez Grana ${ }^{1,2}$, J. Meaburn ${ }^{3}$ and \\ Eduardo de la Fuente ${ }^{1,2,4}$ \\ ${ }^{1}$ Instituto de Astronomía y Meteorología, Universidad de Guadalajara, Guadalajara, Jalisco, \\ México email: snk@astro.iam.udg.mx \\ ${ }^{2}$ Departmento de Física, CUCEI, Universidad de Guadalajara, Guadalajara, Jalisco, México \\ ${ }^{3}$ Jodrell Bank Observatory, School of Physics and Astronomy, University of Manchester, \\ United Kingdom \\ ${ }^{4}$ Instituto Nacional de Astrofísica, Optica y Electrónica, Tonantzintla, Puebla, México
}

\begin{abstract}
We present images of a number of lenticular galaxies which appear to be undergoing evolutionary processes such as ram-pressure stripping or gravitational interactions, or possess features indicative of some evolutionary process in the recent past, such as fossil spiral arms, a polar ring-like structure, or a warped disk with box-peanut bulge. All the galaxies were originally identified on digitally co-added photographic plates or films from the UK Schmidt Telescope, showing that such material is still useful in spite of the modern digital surveys available. In some cases CCD follow up is presented.
\end{abstract}

Keywords. galaxies: elliptical and lenticular, $\mathrm{cD}$, galaxies: evolution, galaxies: bulges, galaxies: interactions

NGC 4620 has been classified as a dS0 and is also known as VCC 1902. The DSS image (not shown) does not show any distinguishing features. High contrast images (not shown) from a stack of 13 digitally co-added films of Virgo NE (main results reported in Katsiyannis et al. 1998) show faint 'fossil' spiral arms. These can also be seen in an SDSS image included in Lisker et al. (2006). These authors classify this amd a few similar objects as 'dwarflike S0/Sa' objects. The images shown in Fig. 1 come from CCD images taken with the SPM 2.1m in April 2005. The faint spiral arms can be clearly seen in Fig. 1a. and can be traced for complete turns in a logarithmic scale image (not shown). There seems to be evidence for a ring with diameter of about $1.5 \mathrm{arcmin}$, seen in the residual image (Fig. 1b), and showing bluer colours in a $B-V$ colour map (not shown). The small bulge region is also bluer. The spiral arms seem to start from this ring and have redder colours.

WMMA 196 is an S0 galaxy which was first identified on the stack of 8 digitally coadded $B(J)$ Schmidt plates including the IC 4296 cluster (main results reported in Kemp $\&$ Meaburn (1993)). To the E of the galaxy an arc of low surface brightness emission is seen, and this is also present on the AAT CCD image (Fig. 2a). A bright star nearby prevents us knowing if this arc continues to the $\mathrm{W}$ of the galaxy. This feature may be related to the 'polar rings' seen around many S0 galaxies. In a $B-V$ colour map the disk and bulge are seen to be very red (1.1-1.5) while the arc has bluer $B-V$ colours of $0.6-0-8$, indicating that much star formation may be present in the arc.

ESO 383-05 was identified from the stack of Schmidt plates in Kemp \& Meaburn (1993). It is an edge-on S0/Sa with a large box-peanut type bulge, and a extensive warped disk, which deviates up to 15 degrees from the major axis of the galaxy, and appears to change direction in the SE half of the disk, appearing to bend to the $\mathrm{N}$ initially before 

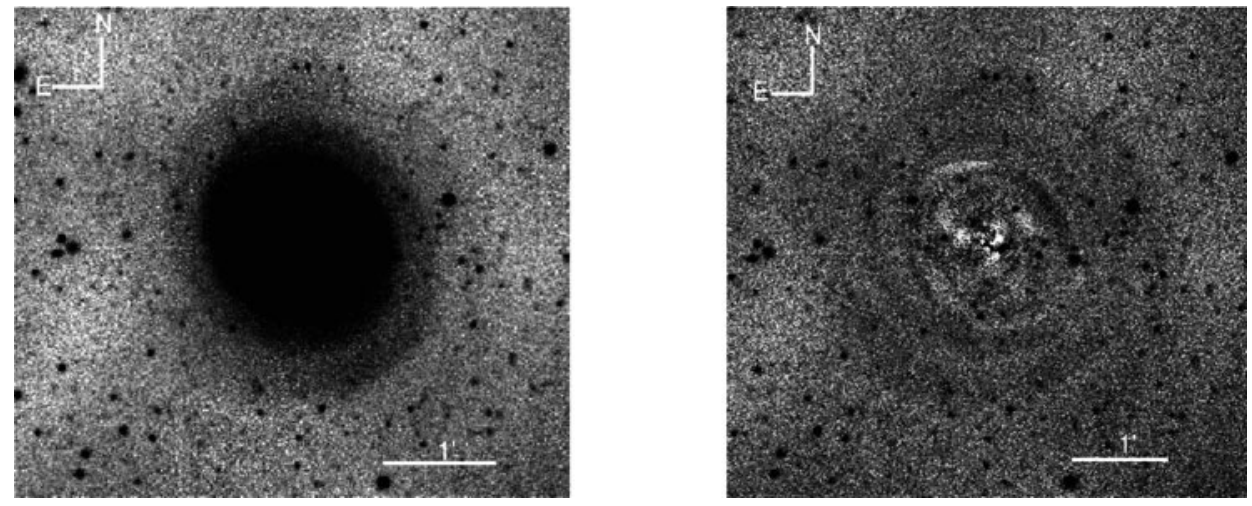

Figure 1. NGC 4620 a) Deep CCD $B$ image at high contrast showing the faint spiral arms b) Residual image in $B$ (after subtracting isophotal model) showing arms and ring
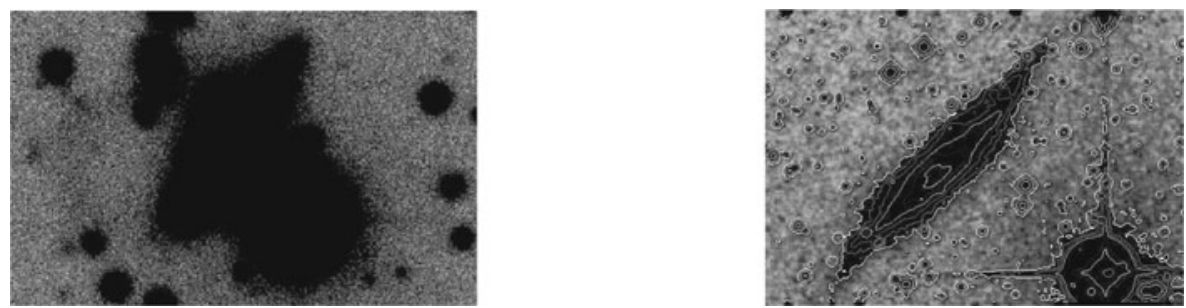

Figure 2. a) WMMA 196: deep image in $B$ showing arc to east of galaxy (and bright star to SW) b) ESO 383-05: isophotes superimposed on deep image from 8 digitally co-added $B(J)$ Schmidt plates, showing warped disk and large box-peanut bulge

bending to the S further out (Fig. 2b). Lütticke et al. (2004) include it in a sample of galaxies with a Thick Boxy Bulge.

ESO 383-45: At low contrast this appears as a normal-looking S0 galaxy, with disk and bulge, but at high contrast a very extended halo is seen, as well as several filments directed away from the A3565 cluster center (Schmidt images in Kemp \& Meaburn 1993, CCD and Schmidt follow-up in Kemp et al. 2005). The extended halo may indicate that this galaxy is a merger product formed after a gravitational interaction between two disk galaxies (Naab \& Burkert 2003), while the filaments may be material ram-pressurestripped from the galaxy by the dense IGM, if the galaxy is moving towards IC 4296 and the cluster centre (Kemp et al. 2005), or they may be tidal filaments composed of stars.

\section{Acknowledgements}

SNK wishes to thank PROMEP for financial help. Pérez Grana acknowledges support from CONACyT (México) grant 193057. E de la F acknowledges support from CONACyT (México) grant 124449 and SNI III 1326.

\section{References}

Katsiyannis, A. C., Kemp, S. N., Berry, D. S., \& Meaburn, J. 1998, A\&AS 132, 387

Kemp, S. N. \& Meaburn, J. 1993, A\&A 274, 19

Kemp, S. N., de la Fuente, E., Franco-Balderas, A., \& Meaburn, J. 2005, ApJ 624, 680

Lisker, T., Grebel, E. K., \& Binggeli, B. 2006, AJ 132, 497

Lütticke, R., Pohlen, M., \& Dettmar, R. -J. 2004, A\&SA 417, 527

Naab, T. \& Burkert, A. 2003, ApJ 597, 893 